Artículo científico

Volumen 30(2):353-365. Mayo-agosto, 2019

e-ISSN 2215-3608, doi:10.15517/am.v30i2.34032

https://revistas.ucr.ac.cr/index.php/agromeso/index

\title{
Estructura e impacto de la diversidad taxonómica en cacao del Soconusco, Chiapas, México ${ }^{1}$
}

\section{Structure and impact of taxonomic diversity on cocoa of Soconusco, Chiapas, México}

\author{
Gicli Manuel Suárez-Venero ${ }^{2}$, Carlos Hugo Avendaño-Arrazate ${ }^{3}$, Pablo Amín Ruiz-Cruz \\ Paulina Estrada-de-los-Santos ${ }^{4}$
}

1 Recepción: 18 de julio, 2018. Aceptación: 25 de octubre, 2018. Este trabajo formó parte de una investigación desarrollada del proyecto: "Tecnología para la producción sostenible de Theobroma cacao L. con materiales genéticos compatibles a los cambios climáticos", de la estancia posdoctoral del primer autor por medio de la Agencia Mexicana de Cooperación Internacional para el Desarrollo (AMEXCID) de la Secretaría de Relaciones Exteriores (SRE) de México.

2 Universidad de Guantánamo. Avenida Che Guevara, km 1.5 Carretera a Jamaica, Guantánamo, CP. 95 100, Cuba. gicli@cug.co.cu (https:// orcid.org/0000-0002-5235-9192).

3 Instituto Nacional de Investigaciones Forestales Agrícolas y Pecuarias (INIFAP). Campo Experimental Rosario Izapa, Km. 18 Carretera Tapachula - Cacahoatán, Tuxtla Chico, CP. 30870, Chiapas, México. avendano.carlos@inifap.gob.mx (autor para correspondencia; https:// orcid.org/0000-0003-3591-2280), ruiz.amin@inifap.gob.mx

4 Instituto Politécnico Nacional, Escuela Nacional de Ciencias Biológicas. Prolongación de Carpio y Plan de Ayala s/n. Colonia Santo Tomás. Delegación Miguel Hidalgo. Ciudad de México, México.CP. 11340.pestradadelossantos@gmail.com

\section{Resumen}

Introducción. El cultivo del cacao en México constituye un rubro exportable que garantiza la subsistencia de miles de familias. El estado de Chiapas es uno de los productores más importantes; sin embargo, aún los rendimientos son bajos, debido a diferentes factores que pueden ser minimizados con un adecuado diseño agroforestal que responda a los requerimientos del cultivo. Objetivo. El objetivo de este trabajo fue evaluar la estructura de la diversidad taxonómica en plantaciones de cacao y analizar su influencia sobre el cultivo en el Soconusco, Chiapas, México. Materiales y métodos. La investigación se efectuó durante los meses de enero a mayo del 2018, en trece municipios: Cacahoatán, Tuxtla Chico, Frontera Hidalgo, Metapa, Suchiate, Tapachula, Huixtla, Tuzantán, Huehuetán, Escuintla, Acacoyagua, Acapetagua y Mapastepec. Se establecieron parcelas representativas de 50x20 m, en las que se contabilizaron y clasificaron taxonómicamente las especies presentes. Se evaluó el diámetro a altura de pecho (DAP) y la altura total $(\mathrm{Ht})$. Se realizó un análisis para definir los estratos (inferior, medio y superior) en la estructura vertical del sistema agroforestal y se hicieron consideraciones sobre la influencia de los resultados en el cacao. Resultados. Se determinaron 35 especies arbóreas con 199 plantas que pertenecen a veintidós familias. Las especies más importantes fueron Cordia alliodora, Tabebuia rosea y Pouteria sapota. Conclusión. Las especies de árboles de sombra registradas en la estructura vertical y horizontal de los sistemas agroforestales de cacao de la región del Soconusco, Chiapas, generaron un exceso de sombra equivalente a una media en la región de 14,16 \% de la iluminación total, y la consecuente competencia por la luz entre las especies estudiadas, incluyendo el cultivo del cacao.

Palabras clave: sistema agroforestal, familia, árbol, inventario. 


\begin{abstract}
Introduction. The cultivation of cocoa in Mexico is an exportable item that guarantees the subsistence of thousand families. The state of Chiapas is one of the most important producers; however, even the yields are low due to different factors. These can be minimized with appropriate agroforestry design that responds to the requirements of the crop. Objective. The objective of this work was to evaluate the structure of taxonomic diversity in cocoa plantations, and analyze its influence on the crop in Soconusco, Chiapas, Mexico. Materials and methods. The research was carried out from January to May 2018 in thirteen municipalities: Cacahoatán, Tuxtla Chico, Frontera Hidalgo, Metapa, Suchiate, Tapachula, Huixtla, Tuzantán, Huehuetán, Escuintla, Acacoyagua, Acapetagua and Mapastepec. Representative plots of 50x20 m were established, in which the present species were counted and classified taxonomically. The diameter at chest height (DAP) and total height (Ht) were evaluated. An analysis was made to define the strata (lower, middle and upper) in the vertical structure of the agroforestry system, and considerations were made about the influence of the results on the cocoa. Results. 35 tree species were determined with 199 plants belonging to twenty-two families. The most important species were Cordia alliodora, Tabebuia rosea and Pouteria sapota. Conclusion. The shade tree species registered in the vertical and horizontal structure of the agroforestry cocoa systems of the Soconusco region, Chiapas, generated an excess of shade equivalent to an average in the region of $14.16 \%$ of the total illumination, and the consequent competition for light among the species studied, including cocoa cultivation.
\end{abstract}

Keywords: agroforestry system, family, tree, inventory.

\title{
Introducción
}

El cultivo del cacao (Theobroma cacao L.) en México constituye un rubro exportable importante que garantiza la subsistencia de miles de familias. México ocupa el octavo lugar en la producción de este cultivo a nivel mundial, pero en los últimos diez años se ha observado una tendencia decreciente en su producción. A pesar de esto, la producción de cacao avanzó ligeramente en 2017 (27 326 t) con respecto al 2016 (26 969 t) (SIAP, 2018).

Chiapas es uno de los estados productores de cacao más importantes en México y ocupa el segundo lugar en producción después de Tabasco. Al cierre del 2017 tuvo una superficie destinada al cultivo de 17812 ha, con una producción media de 9707 t e intención de cosecha en el año 2018 de 9675 t, traducibles en un rendimiento promedio de 0,44 t.ha-1 $($ SIAP, 2018). Las principales regiones de producción son Norte, Centro, Soconusco y Selva-Norte. Son muchos los factores que intervienen en estos resultados productivos; entre ellos el aspecto fitosanitario y los actuales cambios climáticos que constituyen retos fundamentales. No obstante, ambos factores se pueden minimizar con un adecuado manejo de las plantaciones, que considere un correcto diseño agroforestal, en que la estructura horizontal y vertical responda a los requerimientos del cultivo y logre la conservación de los recursos naturales, suelo y agua.

La mayor parte de las plantaciones de cacao en el mundo se encuentran establecidas en convivencia con especies de árboles de sombra, ya sea bajo un diseño de manejo agroforestal o no. Esta convivencia se asemeja al ambiente de origen del cacao, pero con manejo de las especies de sombra con el objetivo de obtener mayor productividad bajo esas condiciones. En este contexto Almeida y Valle (2007) sugirieron cultivar el cacao bajo sombra tras realizar experimentos de tolerancia a altas radiaciones.

La función que cumplen las especies de sombra en las plantaciones de cacao, ha sido estudiada por Somarriba y Quesada (2005) y Silva et al. (2013). Aunque la sombra tiene grandes ventajas para el cultivo (González, 2008), su exceso puede afectarlo considerablemente (Alvim et al., 1974; Beer et al., 2003). Debido a esto, es necesaria una adecuada selección de las especies para sombra en los sistemas agroforestales de cacao. En México se han 
desarrollado algunas investigaciones sobre este tema; Ramírez et al. (2013) en el estado Tabasco y Salgado-Mora et al. (2007) y Roa et al. (2009) en el Soconusco, Chiapas. Estos últimos autores indicaron que al no tener ningún registro de la estructura y función de los árboles que son utilizados como sombra para el cultivo en la región del Soconusco, se complica el desarrollo de políticas, proyectos o investigaciones que lleven al mejoramiento del cultivo y al conocimiento de los recursos naturales con los que se cuenta en esta región del sur del estado de Chiapas.

Conocer la estructura e influencia que poseen las especies de sombra sobre el cultivo, permite adoptar estrategias con enfoque ecológico y agronómico en función de mejorar la sostenibilidad de estos sistemas agroforestales. Por ello, el objetivo de este trabajo fue evaluar la estructura de la diversidad taxonómica en plantaciones de cacao y analizar su influencia sobre el cultivo del cacao en el Soconusco, Chiapas, México.

\section{Materiales y Métodos}

\section{Descripción del área de estudio}

La investigación se desarrolló durante los meses de marzo a mayo de 2018 en trece municipios de Soconusco, sur del estado de Chiapas, México. La región de Soconusco está localizada entre las coordenadas $15^{\circ} 19^{\prime} \mathrm{N}$ y $92^{\circ} 44^{\prime} \mathrm{W}$, cubre $4605,4 \mathrm{~km}^{2}$, lo que representa $6,28 \%$ de la superficie estatal, y es la séptima región de mayor extensión territorial en el estado de Chiapas.

La región se caracteriza por climas cálidos y semicálidos; predomina el cálido subhúmedo con lluvias de verano, seguido por el cálido húmedo con lluvias abundantes de verano. Por tanto, de acuerdo con la clasificación de Köppen modificada por García (1973), el clima predominante en la región de estudio es del tipo Aw2(w)ig con humedad relativa media de $79,4 \%$, con la temperatura media anual de $26,8{ }^{\circ} \mathrm{C}$ (CNA, 1998). Presenta diez unidades de suelo, las principales son cambisol, acrisol y feozem (IUSS, 2008). En las zonas de producción de cacao, por lo general, los suelos predominantes son el andosol y fluvisol (López et al., 1988).

\section{Muestreo}

Para determinar las zonas de muestreo, se realizó previamente un recorrido exploratorio por el $90 \%$ de las plantaciones de cacao en cada municipio que desarrolla el cultivo del cacao en el Soconusco, Chiapas, con el objetivo de definir criterios para seleccionar municipios y parcelas experimentales objeto del inventario, en conjunto con los productores y decisores de cada zona.

Se realizaron entrevistas y diálogos con los productores de cacao, lo que permitió definir los siguientes criterios de selección: 1) superficie plantada con el cultivo y su representación con el total del estado; 2) número de productores dedicados a la actividad cacaotera y su representación con el total del estado; 3) porcentaje del aporte a la producción total del estado; 4) representatividad geográfica por niveles de altura desde 0-800 m. Con base en los criterios anteriores se seleccionaron trece de los quince municipios que integran la región del Soconusco, Chiapas (Cuadro 1).

\section{Inventario forestal en el sistema agroforestal con cacao}

El muestreo se realizó en parcelas de 20 x 50 m en cada uno de los municipios, donde se cuantificó el número de árboles de cacao y se realizó el inventario de las especies presentes. A cada árbol se le tomó el nombre común, que permitió la identificación taxonómica del componente arbóreo (familia, género y especie), con base en el manual de árboles de Centroamérica del CATIE (Barrance et al., 2003) y árboles tropicales de México (Pennington y Sarukhán, 2005). A cada árbol se le midió el área basal, la cual se obtuvo a partir de la variable de diámetro 
Cuadro 1. Localidades para el inventario forestal en el sistema agroforestal con cacao en la región de Soconusco, Chiapas, México. 2018.

Table 1. Locations for the forest inventory in the agroforestry system with cocoa in the Soconusco region, Chiapas, Mexico. 2018.

\begin{tabular}{|c|c|c|c|c|c|c|}
\hline Municipio & Localidad & Propietario & $\begin{array}{c}\text { Edad del cultivo } \\
\text { (años) }\end{array}$ & Coord. X & Coord. Y & Altitud (m) \\
\hline Acacoyagua & Los cacaos & Adelita Miranda Paz & 5 & 536672 & 1701221 & 398 \\
\hline Acapetagua & Rancho San Antonio & $\begin{array}{l}\text { Alberto Montes } \\
\text { Espinoza }\end{array}$ & 30 & 530773 & 1692492 & 34 \\
\hline Escuintla & El triunfo & $\begin{array}{c}\text { Gabriel Escobar } \\
\text { Mejía }\end{array}$ & 6 & 544935 & 1697062 & 36 \\
\hline Frontera Hidalgo & La primavera & $\begin{array}{l}\text { Santiago Galindo } \\
\text { Vázquez }\end{array}$ & 9 & 587577 & 1635447 & 83 \\
\hline Huehuetán & Rancho Esquipulas & $\begin{array}{c}\text { Transito Cruz } \\
\text { Guzmán }\end{array}$ & 12 & 559941 & 1657255 & 17 \\
\hline Huixtla & $\mathrm{El}$ arenal & Efraín Santiago Cruz & 10 & 554524 & 1666228 & 19 \\
\hline Mapastepec & La fronterita & $\begin{array}{c}\text { Emilio Marroquín } \\
\text { Hernández }\end{array}$ & 25 & 514271 & 1700423 & 29 \\
\hline Metapa & Los cacahuatales & $\begin{array}{c}\text { Francisca Hernández } \\
\text { Guzmán }\end{array}$ & 30 & 586045 & 1637418 & 91 \\
\hline Suchiate & $\begin{array}{l}\text { Manuel Ávila } \\
\text { Camacho }\end{array}$ & $\begin{array}{c}\text { Manuel Mérida } \\
\text { Villatoro }\end{array}$ & 20 & 582086 & 1618980 & 19 \\
\hline Tapachula & Raymundo Enriquez & Eder Herrera López & 20 & 571924 & 1643444 & 60 \\
\hline Tuxtla Chico & C. E. Rosario Izapa & Rosario Izapa & 7 & 590658 & 1655363 & 433 \\
\hline Tuzantán & Tercer cantón & Olinto López Jiménez & 25 & 558966 & 1669483 & 33 \\
\hline Cacahoatán & Santa Martha & $\begin{array}{c}\text { Anselmo } \\
\text { Mounguerza }\end{array}$ & 30 & 586167 & 1658130 & 500 \\
\hline
\end{tabular}

medido a altura de pecho (DAP: 1,3 m sobre el nivel del suelo); se midió con una cinta diamétrica de $5 \mathrm{~m}$ (Jackson, MS). Además, se midió la altura con un clinómetro.

\section{Variables de respuesta}

\section{Estructura horizontal}

Se calculó el índice de valor de importancia (IVI) según Curtis y Mcintosh (1951), que determina las especies más importantes en el uso específico de la tierra. Para ello, se utilizaron las siguientes fórmulas:

$I V I=$ densidad relativa + dominancia relativa + frecuencia relativa

Donde:

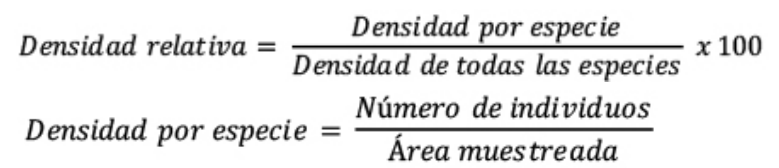




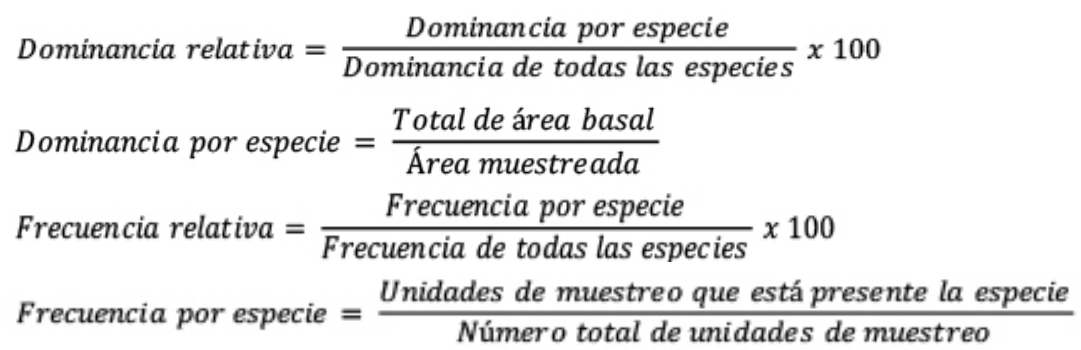

\section{Estructura vertical}

Se evaluó la estratificación vertical dada por la distribución de las copas en el perfil vertical respecto al cultivo del cacao, en cada uno de los municipios muestreados. Se identificaron las especies presentes en los estratos bajo, medio, alto. Los estratos se clasificaron en correspondencia con la altura de las especies de sombra según Somarriba et al. (2004), como sigue:

a) Estrato inferior: especies que ocupan una altura entre 2-8 $\mathrm{m}$.

b) Estrato medio: especies que ocupan una altura entre 9-25 $\mathrm{m}$.

c) Estrato superior: especies que ocupan una altura entre $26-40 \mathrm{~m}$.

\section{Influencia de la estructura arbórea sobre el cultivo del cacao en el sistema agroforestal}

Se consideraron los resultados de las estructuras horizontal y vertical en función del cultivo principal del sistema agroforestal. Se contó el número de plantas de cacao en cada parcela y las existentes en la zona bajo el dosel de sombra. Se realizó un análisis en correspondencia con los requerimientos de sombra del cultivo y se determinó la capacidad de sombra del conjunto de doseles de los árboles en las parcelas, a partir del porcentaje de iluminación promedio que penetra en el interior de la plantación, medido por la cantidad de luxes (lux.FC-1). Para aleatorizar el dato de la parcela, se realizaron veinte repeticiones repartidas aleatoriamente dentro de cada una de ellas, cuyos resultados se promediaron por parcela y por municipio. Para la medición se utilizó un luxómetro marca SPER SCIENTIFIC 840020.

\section{Análisis estadístico}

Los datos se sistematizaron y registraron en una base de datos creada con el paquete de Excel versión 9.1. Se calculó la frecuencia y el promedio de especies. Además, se realizó un análisis de varianza y una prueba de comparación de medias para la variable cantidad de luz con el paquete estadístico STATGRAPHICS plus ver 5.1.

\section{Resultados}

\section{Estructura horizontal}

En el Soconusco, se identificaron 35 especies arbóreas que se seleccionaron por los productores de cacao como sombra de este cultivo. Se trata de la vegetación nativa del bosque natural y vegetación secundaria por regeneración natural (Cuadro 2). 
Cuadro 2. Catálogo de especies sombreadoras de cacao encontradas en los municipios muestreados del Soconusco, Chiapas, México. 2018.

Table 2. Catalog of cocoa shading species found in the sampled municipalities of Soconusco, Chiapas, Mexico. 2018.

\begin{tabular}{|c|c|c|c|}
\hline Familia & Especie & Nombre común & Usos \\
\hline Anacardiaceae & Mangifera indica $\mathrm{L}$ & Mango & Frutal \\
\hline Apocynaceae & Aspidosperma megalocarpon Müll.Arg & Chiche & Maderable \\
\hline Apocynaceae & Stemmadenia donnell-smithii (Rose) Woodson & Chapon & Maderable \\
\hline Bignoniaceae & Tabebuia donnell-smithii Rose & Primavera & Maderable \\
\hline Bignoniaceae & Tabebuia rosea (Bertol) DC. & Roble & Maderable \\
\hline Boraginaceae & Cordia alliodora (Ruiz\& Pav.) Oken & Laurel & Maderable \\
\hline Bombacaceae & Ceiba pentandra (L.) Gaertn. & Ceiba & Maderable \\
\hline Burseraceae & Bursera simaruba (L.) Sarg. & Jiote & Maderable \\
\hline Cecropiaceae & Cecropia obtusifolia Bertol & Guarumo & Otros usos \\
\hline Chrysobalanaceae & Couepia polyandra (Kunth) Rose & Carnero & Maderable \\
\hline Combretaceae & Terminalia amazonia (J.F.Gmel.) Exell & Guayabo volador & Maderable \\
\hline Clusiaceae & Garcinia humilis (Vahl) C.D. Adams & Achachayru & Frutal \\
\hline Euphorbiaceae & Sapium macrocarpum Müll. Arg & Chonte & Maderable \\
\hline Euphorbiaceae & Hippomane mancinella $\mathrm{L}$. & Manzanillo & Maderable \\
\hline Fabaceae & Inga micheliana Harms & Chalum & Maderable \\
\hline Fabaceae & Schizolobium parahyba Müll. Arg & Zope & Maderable \\
\hline Fabaceae & Poeppigia procera C. Presl & Tepemistle & Maderable \\
\hline Fabaceae & Erythrina fusca Lour & Pito & Otros usos \\
\hline Lauraceae & Persea americana Mill. & Aguacate & Frutal \\
\hline Malvaceae & Chiranthodendron pentadactylon Larreat & Canaque & Maderable \\
\hline Malvaceae & Theobroma bicolor Humb. \& Bonpl & Pataste & Frutal \\
\hline Meliaceae & Cedrela odorata $\mathrm{L}$ & Cedro & Maderable \\
\hline Meliaceae & Guarea glabra Vahl. & Cedrillo & Maderable \\
\hline Moraceae & Castilla elastica Sesse & Hule & Maderable \\
\hline Moraceae & Ficus sp. & Mata Palo & Otros usos \\
\hline Moraceae & Maclura tinctoria (L.) Steud & Mora Blanco & Maderable \\
\hline Muntingiaceae & Muntingia calabura L. & Capulín & Otros usos \\
\hline Rutaceae & Citrus nobilis Lour & Mandarina & Frutal \\
\hline Rutaceae & Citrus sinensis L. & Naranja & Frutal \\
\hline Rutaceae & Citrus aurantifolia (Christm) Swingle & Limón & Frutal \\
\hline Sapindaceae & Nephelium lappaceum $\mathrm{L}$. & Rambután & Frutal \\
\hline Sapotaceae & Manilkara zapota (L.) van Royen & Chicozapote & Frutal \\
\hline Sapotaceae & Pouteria sapota (Jacq.) H. E. Moore \& Stearn & Zapote & Frutal \\
\hline Sterculiaceae & Sterculia apelata (Jacq.) Karst & Castaño & Maderable \\
\hline Tiliaceae & Apeiba tibourbou Aubl. & Peine de Mico & Maderable \\
\hline
\end{tabular}

Las especies registradas, se agruparon en 32 géneros y 22 familias. Los géneros más representados fueron Citrus $(8,5 \%)$ y Tabebuia $(5,71 \%)$, que en su conjunto agruparon a un $25,12 \%$ de los árboles. Las familias más representadas fueron Fabaceae, Moraceae, y Rutacea, con cuatro, tres y tres especies cada una, respectivamente; seguidas de las familias Apocynaceae, Bignosacae, Euphorbiaceae, Malvaceae, Meliaceae y Sapotaceae, con dos especies cada una. Estas familias agruparon el $54,1 \%$ de las especies inventariadas. 
La mayor diversidad se registró en los municipios de Tuxtla Chico y Escuintla, con doce especies cada uno, seguidos por Acacoyagua y Acapetagua con once y ocho especies, respectivamente. En Tapachula y Huehuetán se inventarió el menor número de especies con una y dos, respectivamente.

Respecto a la abundancia de especies, el inventario determinó un total de 199 árboles. El C. alliodora, T. rosea y P. sapota fueron las especies más abundantes, con 29, 26 y 23 árboles, respectivamente (Figura 1).

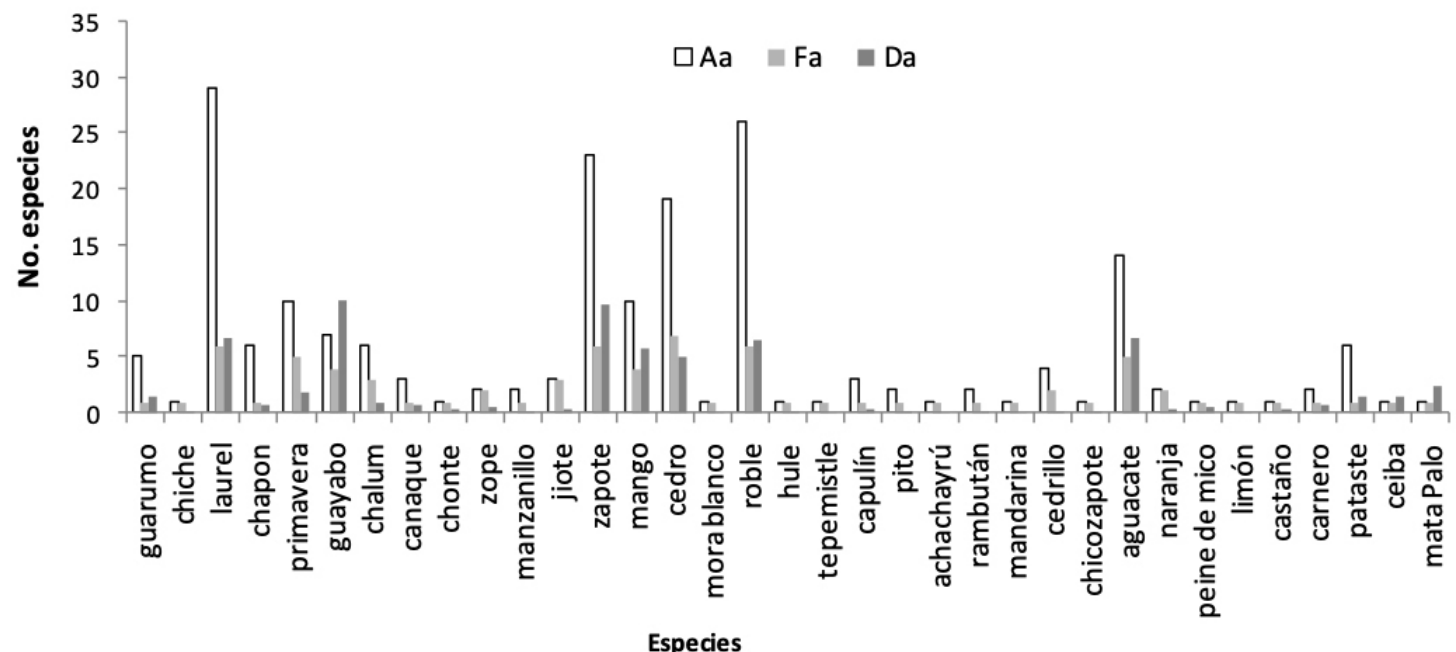

Figura 1. Abundancia absoluta (Aa), frecuencia absoluta (Fa) y dominancia absoluta (Da) de las especies de árboles de sombra registradas en el inventario en todas las parcelas muestreadas de cacao en el Soconusco, Chiapas, México. 2018.

Figure 1. Absolute abundance ( $\mathrm{Aa}$ ), absolute frequency (Fa), and absolute dominance ( $\mathrm{Da}$ ) of the shade tree species registered in the inventory in all the plots sampled of cocoa in the Soconusco, Chiapas, Mexico. 2018.

La especie C. alliodora mayormente se encontró en la parcela de Frontera Hidalgo (diecisiete árboles), el $T$. rosea en el municipio Tuzantán (nueve árboles) y el P. sapota en Tapachula (ocho árboles). Coincidentemente, estas especies resultaron ser las más importantes por su densidad, frecuencia y dominancia relativa (Figura 2).

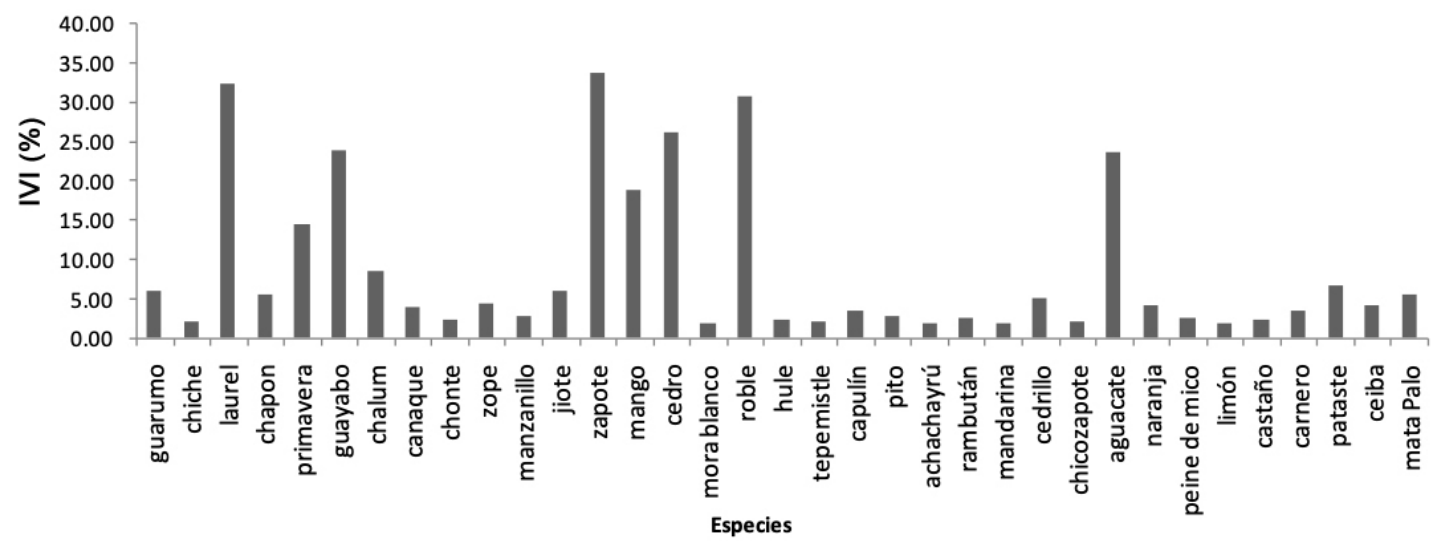

Figura 2. Índice de valor de importancia (IVI) de las especies inventariadas en plantaciones de cacao en la región del Soconusco, Chiapas, México. 2018.

Figure 2. Importance value index (IVI) of the species inventoried in cocoa plantations in the Soconusco region, Chiapas, Mexico. 2018. 
La densidad de especies de árboles de sombra en todos los municipios varió entre 80 y 300 árboles.ha $^{-1}$. Los municipios con mayor densidad de especies de sombra en el interior de las plantaciones de cacao, fueron Tuxtla Chico, Tuzantán y F. Hidalgo, con valores entre 230 y 300 árboles.ha $^{-1}$, no así en las plantaciones de Cacahoatán, cuya densidad solo fue de 40 árboles.ha-1 ${ }^{-1}$, siendo este el único registro de densidad adecuada para el cultivo.

\section{Estructura vertical}

El $73 \%$ y $75 \%$ del total de árboles y especies registrados, respectivamente, se encontraron ocupando el estrato medio de las plantaciones de cacao en todos los municipios, siendo C. alliodora, $P$. sapota y $T$. rosea, las especies más abundantes en ese nivel, mientras que, los municipios con mayor número de árboles fueron Tuxtla Chico, Frontera Hidalgo y Tuzantán (Cuadro 3).

Cuadro 3. Número de especies de árboles de sombra registrados por estratos en parcelas de cacao en los municipios estudiados del Soconusco, Chiapas, México. 2018.

Table 3. Number of shade tree species registered by strata in the cacao plots in the studied municipalities of Soconusco, Chiapas, Mexico. 2018.

\begin{tabular}{|c|c|c|c|c|c|c|}
\hline \multirow[b]{2}{*}{ Municipios } & \multicolumn{2}{|c|}{ Estrato inferior } & \multicolumn{2}{|c|}{ Estrato medio } & \multicolumn{2}{|c|}{ Estrato superior } \\
\hline & No. especies & $\%$ del total & No. especies & $\%$ del total & No. especies & $\%$ del total \\
\hline Cacahoatán & 0 & 0,00 & 0 & 0,00 & 4 & 22,22 \\
\hline Tuxtla Chico & 5 & 13,89 & 24 & 16,55 & 1 & 5,56 \\
\hline F. Hidalgo & 3 & 8,33 & 17 & 11,72 & 3 & 16,67 \\
\hline Metapa & 3 & 8,33 & 10 & 6,90 & 3 & 16,67 \\
\hline Suchiate & 0 & 0,00 & 12 & 8,28 & 1 & 5,56 \\
\hline Tapachula & 1 & 2,78 & 7 & 4,83 & 0 & 0,00 \\
\hline Huixtla & 3 & 8,33 & 10 & 6,90 & 2 & 11,11 \\
\hline Tuzantán & 6 & 16,67 & 16 & 11,03 & 0 & 0,00 \\
\hline Huehuetán & 1 & 2,78 & 6 & 4,14 & 0 & 0,00 \\
\hline Excuintla & 7 & 19,44 & 14 & 9,66 & 0 & 0,00 \\
\hline Acacoyagua & 6 & 16,67 & 7 & 4,83 & 4 & 22,22 \\
\hline Acapetagua & 1 & 2,78 & 13 & 8,97 & 0 & 0,00 \\
\hline Mapastepec & 0 & 0,00 & 9 & 6,21 & 0 & 0,00 \\
\hline Total & 36 & 100 & 145 & 100 & 18 & 100 \\
\hline
\end{tabular}

El menor número de especies se registró en el nivel superior, donde P. americana y $M$. indica fueron las especies de mayor presencia, con el mayor número de árboles registrado en los municipios Acacoyagua y Cacahoatán.

Por lo general, las plantas de cacao en todas las plantaciones muestreadas se encontraron en el estrato inferior, compartiendo el mismo espacio vertical con un $18 \%$ y $50 \%$ del total de árboles y especies, respectivamente. Lo anterior significa que, en los actuales sistemas agroforestales con cacao del estado, se encuentran especies en similares alturas con el cultivo, tales como: S. donnell-smithii, T. abebuia, C. odorata y Guarea glabra, entre otras. En los municipios de Cacahoatán, Suchiate y Mapastepec no se registraron especies en este estrato. 


\section{Influencia de la morfología de árboles de sombra sobre el cultivo del cacao}

En las plantaciones de cacao de los municipios Acacoyagua y Tapachula, se registraron los mayores valores de iluminación de los árboles de sombra, seguidos por Escuintla, Suchiate y Mapastepec. Sin embargo, estos fueron muy bajos, comparados con los valores de iluminación promedio, óptimos para el cultivo del cacao. La cantidad de iluminación evaluada en el interior de las parcelas (Cuadro 4), mostró que los valores máximos fueron muy diferentes entre las plantaciones en los municipios.

Cuadro 4. Iluminación medida en el interior de las parcelas estudiadas en trece municipios del Soconusco, Chiapas, México. 2018.

Table 4. Lighting measured inside the plots studied in thirteen municipalities of Soconusco, Chiapas, Mexico. 2018.

\begin{tabular}{|c|c|c|c|c|c|}
\hline $\begin{array}{l}\text { Municipios/ } \\
\text { Estadígrafos }\end{array}$ & Media & $\begin{array}{c}\text { Valor Mínimo } \\
\left.\text { (lux.FC }{ }^{-1}\right)\end{array}$ & $\begin{array}{c}\text { Valor Máximo } \\
(\text { lux.FC-1) }\end{array}$ & C. V. $(\%)$ & E.S. \\
\hline Cacahoatán & $2321,55 \mathrm{ab}$ & 510 & 4530 & 54,73 & 284,15 \\
\hline Tuxtla Chico & $1486,00 \mathrm{a}$ & 900 & 2200 & 25,96 & 86,27 \\
\hline Frontera Hidalgo & $1818,65 a b$ & 943 & 2700 & 30,70 & 124,87 \\
\hline Metapa & $4170,00 \mathrm{ab}$ & 940 & 7400 & 48,23 & 449,77 \\
\hline Suchiate & $4630,00 \mathrm{ab}$ & 450 & 8810 & 56,22 & 582,06 \\
\hline Tapachula & $4840,00 \mathrm{~b}$ & 550 & 9400 & 55,54 & 601,12 \\
\hline Huixtla & $4015,50 \mathrm{ab}$ & 1100 & 6700 & 42,76 & 383,94 \\
\hline Tuzantán & $3994,00 \mathrm{ab}$ & 700 & 7250 & 53,60 & 478,74 \\
\hline Huehuetán & $3994,00 \mathrm{~d}$ & 700 & 7250 & 53,60 & 478,74 \\
\hline Escuintla & $4680,50 \mathrm{~b}$ & 500 & 8900 & 52,35 & 547,97 \\
\hline Acacoyagua & $11578,60 \mathrm{c}$ & 826 & 25000 & 67,93 & 1758,87 \\
\hline Acapetagua & $2499,25 \mathrm{ab}$ & 975 & 4200 & 39,27 & 219,51 \\
\hline Mapastepec & $4810,00 \mathrm{~b}$ & 820 & 8800 & 51,65 & 555,60 \\
\hline
\end{tabular}

C.V.: coeficiente de variación; ES: error estándar de la media / C.V.: coefficient of variation; E.S.: standard error of the mean.

Otro de los efectos que se observaron, fue la heterogeneidad de la iluminación dentro de las plantaciones, demostrado por el alto coeficiente de variación en todos los municipios (Cuadro 4), siendo significativos los municipios de Acacoyagua, Tapachula, Suchiate, Cacaohatán, Tuzantán, Huehuetán, Escuintla y Mapastepec, donde se evaluaron más del $50 \%$ de coeficiente de variación.

Los resultados del registro de la densidad de plantas de cacao por parcela, indicaron bajo porcentaje de población en las plantaciones de cacao en todos los municipios, con excepción de Tuxtla Chico; con muy bajas densidades en los municipios de Acapetagua, Escuintla y Mapastepec. De igual forma, se registró, poca cantidad de plantas de cacao bajo los doseles de sombra de diferentes especies, con la mayor influencia de M. indica, P. sapota y P. americana, debido a su inadecuado manejo (Cuadro 5). 
Cuadro 5. Cantidad de plantas de cacao por parcela, en los municipios estudiados del Soconusco, Chiapas, México. 2018.

Table 5. Quantity of cocoa plants per plot, in the studied municipalities of Soconusco, Chiapas, Mexico. 2018.

\begin{tabular}{lccccc}
\hline $\begin{array}{l}\text { Municipios/ } \\
\text { Variables }\end{array}$ & $\begin{array}{c}\text { No. cacao por } \\
\text { parcela }\end{array}$ & Árboles/ha & Población (\%) & $\begin{array}{c}\text { Total plantas } \\
\text { cacao en dosel }\end{array}$ & $\begin{array}{c}\text { Promedio plantas } \\
\text { de cacao por dosel }\end{array}$ \\
\hline Cacahoatán & 68 & 680 & 61,2 & 62 & 15,54 \\
Tuxtla Chico & 108 & 1080 & 97,2 & 285 & 9,50 \\
Frontera Hidalgo & 73 & 730 & 65,7 & 161 & 7,00 \\
Metapa & 55 & 550 & 49,5 & 50 & 3,32 \\
Suchiate & 81 & 610 & 72,9 & 91 & 7,00 \\
Tapachula & 66 & 530 & 49,4 & 38 & 4,20 \\
Huixtla & 53 & 760 & 68,4 & 76 & 3,00 \\
Tuzantán & 76 & 740 & 66,6 & 60 & 3,54 \\
Huehuetán & 74 & 370 & 33.3 & 78 & 7,51 \\
Escuintla & 37 & 520 & 46.8 & 56 & 3,94 \\
Acacoyagua & 52 & 320 & 28,8 & 25 & 4,00 \\
Acapetagua & 32 & 410 & 36,9 & $\mathbf{5 6 , 7 0}$ & 57 \\
Mapastepec & 41 & $\mathbf{6 2 7 , 6 0}$ & & $\mathbf{8 2 , 8 0}$ & 6,32 \\
\hline Promedio & $\mathbf{6 2 , 7 0}$ & & & $\mathbf{5 , 6 0}$ \\
\hline
\end{tabular}

\section{Discusión}

La composición florística determinada fue típica de los sistemas agroforestales con cacao de esta región, con especies comunes de la selva alta perennifolia, como T. amazonia y A. megalocarpon, lo que coincide con las afirmaciones de Roa et al. (2009). Este mismo autor obtuvo resultados similares a los alcanzados en la presente investigación con $P$. sapota en cuatro municipios de esta misma región, donde este fue el frutal más abundante, junto con $M$. indica. Por lo general, el tipo de sombra presente en las plantaciones estudiadas define diversos sistemas de producción familiar, dado la importancia de las especies presentes en los sistemas agroforestales, donde los productores obtienen productos de autoabastecimiento, para el pequeño comercio, para leña (energía) o para buscar opciones de sustituir el cacao con otras especies, en caso de que decidan hacer un cambio de uso del suelo, aspectos que coinciden con lo expresado por Hernández et al. (2015).

Las especies con mayor índice de importancia eran aprovechadas por los productores en estos sistemas agroforestales. En este contexto, C. alliodora era importante en la región por el alto valor de su madera para uso local y combinada con cultivos anuales y perennes en sistemas agroforestales, además de su empleo como planta medicinal en México (Barrance et al., 2003). Por otra parte, T. rosea es un árbol de valor maderero, que se utiliza en los sistemas silvopastoriles, linderos, sombra y ornamental. Sin embargo, P. sapota es un frutal de aceptación en la región, por los aportes que hace al productor a los ingresos económicos familiares. Comúnmente se encuentra formando parte de la estructura arbórea dentro de las plantaciones de café y cacao en muchos países, establecidas o regeneradas naturalmente, ya sea bajo diseño agroforestal o no (Salgado-Mora et al., 2007). Su localización es aleatoria, como se registró en el inventario realizado en el municipio de Tapachula, donde esta fue la única especie registrada con ocho árboles.

De acuerdo con la estructura horizontal y vertical analizada, se evaluó en todos los municipios un exceso de sombra sobre el cultivo, con valores medios máximos de iluminación total entre 4,8 \% y 44,8\%. En este contexto, 
fue determinante la influencia de la densidad de árboles de sombra y su distribución, los cuales fueron muy variables entre los municipios, características típicas de estos tipos de sistemas con regeneración natural de especies y posterior selección por parte del productor.

La densidad de árboles de sombra registrada (80-300 árboles.ha-1 ${ }^{-1}$, fue superior a lo indicado en la tecnología de manejo para el cultivo de cacao y fueron similares a los que obtuvieron Sánchez et al. (2016) en SAFc en el estado de Tabasco, quienes encontraron una densidad media de 286 árboles.ha $^{-1}$, y que varió de 96 a 618 árboles. $\mathrm{ha}^{-1}$. La densidad de siembra para las plantaciones en producción con plantas desarrolladas y autosombreadas, debe ser entre 25 y 50 árboles.ha-1 para originar 60-75 \% de luz total (González, 2008), información que coincide con lo afirmado por Somarriba (2004), quien además afirmó que esta iluminación depende del manejo agronómico del cacao y de las características del dosel de sombra.

La distribución de la iluminación en los sistemas agroforestales estudiados, se observó con una alta variabilidad en las plantaciones de cacao, debido al número y tipo de especies por parcelas, la dimensión del dosel de cada especie y la altura, así como un deficiente manejo de la diversidad de los árboles. Según Silva et al. (2013), si esta diversidad es manejada adecuadamente, se pueden lograr importantes beneficios para el cultivo. Además, las especies de mayor altura y amplios doseles de sombra aportan una densa sombra en mayor cantidad de horas. Al respecto, Somarriba (2002) sugirió que el recorrido sobre el suelo de la sombra de la copa de un árbol, a mayor altura, es más largo que el recorrido de la sombra de la copa de un árbol a baja altura; por ello, Silva et al. (2013) afirmaron que entre los atributos deseables de los árboles de sombra está la altura media entre 15 y $25 \mathrm{~m}$. En el estrato superior de los SAF de cacao en Centroamérica, Ramírez et al. (2013) registraron especies que alcanzaron una altura de $36 \mathrm{~m}$, DAP de $137 \mathrm{~cm}$ y área basal media de $11 \mathrm{~m}^{2} \cdot \mathrm{ha}^{-1}$ a 47,2 $\mathrm{m}^{2}$.ha $\mathrm{h}^{-1}$.

La composición florística evaluada en la estructura vertical y horizontal de los sistemas agroforestales del Soconusco Chiapas, permitió reconocer una competencia por la luz entre los árboles de sombra con el cultivo del cacao, con el consiguiente efecto sobre los procesos fisiológicos que requieren de la iluminación. Por ello, el manejo de la sombra debe ir dirigido a reducir los estreses fisiológicos (Schroth et al., 2000).

Dentro de los procesos que se afectan por el exceso de sombra en el cacao se encuentran la fotosíntesis, la expansión celular de ciertos tejidos y la apertura estomática (Alvim, 1977). Sobre este último proceso, en condiciones normales de iluminación, teóricamente se lleva a cabo durante el periodo del día en que existe mayor iluminación. Sin embargo, después los estomas comienzan a cerrarse por disminución de la intensidad lumínica, por lo que, bajo condiciones de exceso de sombra como están dispuestas las plantaciones de cacao en la región estudiada, este proceso se afecta, y disminuyó además la asimilación de $\mathrm{CO}_{2}$ y el proceso fotosintético, lo cual coincide con lo expresado por Jaimez et al. (2008). Por ello, la fotosíntesis en el cultivo del cacao es mayor sin sombra o con sombra ligera. El exceso de sombra en los sistemas agroforestales de cacao facilita también la aparición de enfermedades como la moniliasis, por la creación de las condiciones de humedad adecuadas para su desarrollo. Para ello, el método más utilizado es el control cultural, que involucra labores para adecuar la sombra y el tamaño del dosel de los árboles de cacao, con lo cual se permite la entrada de luz y el flujo de aire dentro del cultivo (Barros, 1977).

Niveles de iluminación como los obtenidos en este estudio podrían favorecer la reproducción de patógenos, debido a las condiciones microclimáticas originadas en el interior de las plantaciones por el aumento de la humedad relativa. Entre esos patógenos la Moniliophthora roreri fue considerada por Hernández et al. (2015) como el principal factor parasitológico que ha influido en la pérdida de producción y biodiversidad del cacao en Chiapas y se observó en el $100 \%$ de las plantaciones muestreadas en esta investigación.

Por otra parte, la densidad de plantación del cacao es uno de los factores a considerar en el tema de la sombra, por el efecto del autosombreo del cultivo. En general, la densidad de población promedio de las plantaciones de cacao en el estado de Chiapas no superó el 70 \% (630 plantas.ha ${ }^{-1}$ ), sin embargo, la densidad óptima para el cultivo es de 1111 plantas.ha ${ }^{-1}$ y la más empleada en la región estudiada. Lo anterior coincide con lo planteado por ANECACAO (2015) y González et al. (2015). 
Las condiciones de iluminación, puede ser uno de los factores que influye sobre los rendimientos del cultivo en esta región, el cual es en promedio 0,44 t.ha ${ }^{-1}$ (SIAP, 2018). Ello sugiere a los productores adoptar las medidas y decisiones adecuadas en función de implementar labores de rehabilitación, resiembra y manejo de la sombra, considerando el mantenimiento de los frutales, en los municipios de Acacoyagua, Escuintla y F. Hidalgo, por tener una edad que no sobrepasan los diez años. Sin embargo, en el resto de los municipios, donde las plantaciones de cacao son de 20-30 años de edad, se deberán evaluar sistemas de renovación con el consiguiente manejo de la sombra. Para todo tipo de plantación es indispensable lograr el $100 \%$ de población de plantas de cacao.

Estos resultados se relacionan con los reportados en esta misma región por Avendaño et al. (2011), quienes registraron plantaciones de más de veinticinco años. Sin embargo, Hernández et al. (2015) determinaron que las plantaciones de cacao en Chiapas tienen un promedio de 36 años y solo un $4 \%$ de los productores tienen plantaciones nuevas. Estos últimos autores manifestaron que solo el $38 \%$ de los productores en el estado regulan la sombra en las plantaciones de cacao, la gran mayoría indicaron que esta actividad aporta pocos beneficios al cacao e implica un mayor costo de producción, aspecto que explica las condiciones existentes en las plantaciones sobre esta técnica de cultivo.

\section{Conclusión}

Las especies de árboles de sombra registradas en la estructura vertical y horizontal de los sistemas agroforestales de cacao de la región del Soconusco, Chiapas, generan un exceso de sombra equivalente a una media en la región de $14,16 \%$ de la iluminación total, y la consecuente competencia por la luz entre las especies estudiadas, incluyendo el cultivo del cacao.

\section{Literatura citada}

Almeida, A.Y., and R. Valle. 2007. Ecophysiology of the cocoa tree. Braz. J. Plant Phys. 19:425-448. doi:10.1590/S167704202007000400011

Alvim, T. 1977. Cacao. In: P.T. Alvin, and T.T. Kozlowski, editors, Ecophysiology of tropical crops. Academic Press, NY, USA. p. 279-313.

Alvim, T., M.A. Dantas, and F. Vello. 1974. Physiological responses of cacao to environmental factors. Rev. Theobroma 4:3-25.

ANECACAO (Asociación Nacional de Exportadores de Cacao). 2015. Distancia de siembra. ANECACAO, ECU. http://www. anecacao.com/es/servicios/articulos-tecnicos/distancia-de-siembra.html (consultado 10 jul. 2018).

Avendaño, A.C.H., J.M. Villareal, R.E. Campos, R.A. Gallardo, L.A. Mendoza, J.F. Aguirre, A. Sandoval, y S. Espinosa. 2011. Diagnóstico de cacao en México. Universidad Autónoma Chapingo, Texcoco, MEX.

Barrance, A., J. Beer, O.H. Boshier, J. Chamberlain, J. Cordero, G. Detlefsen, B. Finegan, G. Galloway, M. Gómez, J. Gordon, M. Hands, J. Hellin, C. Hughes, M. Ibrahim, R. Leakey, F. Mesén, M. Montero, C. Rivas, E. Somarriba, y J. Stewart. 2003. Árboles de Centroamérica: Un manual para extensionistas. Oxford Forestry Institute, y CATIE, Turrialba, CRI.

Barros, O. 1977. Investigaciones sobre el hongo Monilia roreri Cif. and Par., causante de la pudrición acuosa de la mazorca del cacao; sus daños y su control. Cacaotero Colomb. 3:42-52.

Beer, J., M. Ibrahim, E. Somarriba, A. Barrance, y R. Leakey. 2003. Establecimiento y manejo de árboles en sistemas agroforestales. Oxford Forestry Institut, y CATIE, Turrialba, CRI.

CNA (Comisión Nacional del Agua). 1998. Datos meteorológicos del municipio de Tapachula, Chiapas. CNA, MEX. 
Curtis, J.T., and R.P. Mcintosh. 1951. An upland forest continuum in the prairie-forest border region of Wisconsin. Ecology 32:476-496. doi:10.2307/1931725

García, E. 1973. Modificaciones del sistema de clasificación climática de Köppen para adaptarlo a las condiciones de la República Mexicana. Universidad Nacional Autónoma de México, Ciudad de México, MEX.

González, F. 2008. Ecofisiología del cacao. Blogspot. http://diplomado2007unas.blogspot.com/2008/01/ecofisiologia-del-cacao. html (consultado 20 may. 2018).

González, J., O. Rodríguez, J. De-La-Cruz, y J. Almonte. 2015. Evaluación de tres densidades de plantación de cacao y dos arquitecturas provenientes de reproducción asexual. Rev. APF 4(2):7-12.

Hernández, E., J. Hernández, C.H. Avendaño., G. López, E.R. Garrido, J. Romero, y C. Nava. 2015. Factores socioeconómicos y parasitológicos que limitan la producción del cacao en Chiapas, México. Rev. Mex. Parasitol. 33:232-246.

IUSS (International Union of Soil Science). 2008. Base referencial mundial del recurso suelo. Informes sobre recursos mundiales de suelos 103. FAO, Roma, ITA.

Jaimez, R.E., W. Tezara, I. Coronel, y R. Urich. 2008. Ecofisiología del cacao (Theobroma cacao L.): su manejo en el sistema agroforestal. Sugerencia para su mejoramiento en Venezuela. Rev. For. Venezol. 52:253-258.

López, O., A. Sandoval, y J. Soto. 1988. Sistemas de producción de cacao (Theobroma cacao L.) en la región del Soconusco, Chiapas, México. Secretaría de Agricultura y Recursos Hidráulicos, Ciudad de México, MEX.

Pennington, T.D., y J. Sarukhán. 2005. Árboles tropicales de México: manual para la identificación de las principales especies. Universidad Nacional Autónoma de México, Ciudad de México, MEX.

Ramírez, A., E. García, J.J. Obrador, O. Ruiz, y W. Camacho. 2013. Diversidad florística en plantaciones agroforestales de cacao en Cárdenas, Tabasco, México. Univ. Cienc. 29:215-230.

Roa, H.A., M.G. Salgado, y J. Álvarez. 2009. Análisis de la estructura arbórea del sistema agroforestal de cacao (Theobroma cacao L.) en el Soconusco, Chiapas - México. Acta Biol. Colomb. 14(3):97-110.

Salgado-Mora, M.G., G. Ibarra, J.E. Macías-Sámano, y O. López-Báez. 2007. Diversidad arbórea en cacaotales del Soconusco, Chiapas, México. Interciencia 32:763-768.

Sánchez, F., J. Pérez, O. Obrador, J. José, A. Sol, y O. Ruiz. 2016. Árboles maderables en el sistema agroforestal de cacao en Cárdenas, Tabasco, México. Rev. Mex. Cienc. Agríc. 14:2711-2723. doi:10.29312/remexca.v0i14.440.

SIAP (Servicio de Información Agroalimentaria y Pesquera). 2018. Intención de cosecha de cultivos perennes por estado en México 2018. SIAP, MEX. http://infosiap.siap.gob.mx/opt/agricultura/intension/Intencion_cosechaPerenne_estado2018. pdf (consultado 24 abr. 2018).

Silva, C., L. Orozco, M. Rayment, y E. Somarriba. 2013. Conocimiento local sobre los atributos deseables de los árboles y el manejo del dosel de sombra en los cacaotales de Waslala, Nicaragua. Agrofor. Am. 49:51-60.

Somarriba, E. 2002. Estimación visual de la sombra en cacaotales y cafetales. Agrofor. Am. 9(35-36):86-94.

Somarriba, E. 2004. ¿Cómo evaluar y mejorar el dosel de sombra en cacaotales? Agrofor. Am. 41-42:122-130.

Somarriba, E., C. Harvey, M. Samper, F. Anthony, J. Gonzalez; C. Stracer, and R. Rice. 2004. Conservation of biodiversity in neotropical coffee (Coffea arabica) plantations. In: G. Schroth et al., editors, Agroforestry and boidiversity conservation in tropical landscapes. Island Press, WA, USA. p. 198-226.

Somarriba, E., y F. Quesada. 2005. El diseño y manejo de la sombra en el cacaotal. CATIE, Turrialba, CRI.

Schroth, G., U. Krauss, L. Gasparotto, J.A. Duarte, and K. Vohland. 2000. Pest and diseases in agroforestry systems of the humid tropics. Agroforest. Syst. 50:199-241. doi:10.1023/A:1006468103914 